\title{
Protection of surface states in topological nanoparticles
}

\author{
Gleb Siroki ${ }^{1 *}$, Peter D. Haynes ${ }^{1,2}$, Derek K. K. Lee ${ }^{1}$, and Vincenzo Giannini ${ }^{1,3}$ \\ ${ }^{1}$ Department of Physics, ${ }^{2}$ Department of Materials, Imperial College London and \\ ${ }^{3}$ Instituto de Estructura de la Materia, IEM-CSIC, Madrid, Spain
}

\begin{abstract}
Topological insulators host protected electronic states at their surface. These states show little sensitivity to disorder. For miniaturisation one wants to exploit their robustness at the smallest sizes possible. This is also beneficial for optical applications and catalysis which favour large surface-tovolume ratios. However, it is not known whether discrete states in particles share the protection of their continuous counterparts in large crystals. Here we study the protection of the states hosted by topological insulator nanoparticles. Using both analytical and tight-binding simulations we show that the states benefit from the same level of protection as those on a planar surface. The results hold for many shapes and sustain surface roughness which may be useful in photonics, spectroscopy and chemistry. They complement past studies of large crystals - at the other end of possible length scales. The protection of the nanoparticles suggests that samples of all intermediate sizes also possess protected states.
\end{abstract}

The interest in topological phenomena was triggered by the discovery of previously unknown topological phases - the Quantum Spin Hall effect in 2D and topological insulators (TIs) in 3D [1]. These phases preserve the time-reversal symmetry and occur in some materials with strong spin-orbit coupling. They have been found experimentally $[2,3]$ which triggered the investigation of analogous phenomena in photonic $[4,5]$ and acoustic crystals [6] as well as optical lattices [7]. Electronic TIs host protected surface states at their boundaries. These states are not spin-degenerate so electrons with opposite momenta have opposite spins - see Fig. 1(a). As a result the two backscattering paths (Fig. 1(b)) interfere destructively $[3,8]$. The backscattering is a precursor for localization so its absence means that the states are more robust against localization by non-magnetic impurities and point defects [9] as observed in STM experiments [10]. More generally, the states are protected because the Dirac cone is guaranteed to exist on a TI surface even in the presence of disorder provided the latter is sufficiently smooth. The associated states have non-zero in-plane group velocity and hence cannot be localized. This holds as long as the perturbations (non-magnetic) are weak and do not open a gap in the Dirac cone. The surface states have been previously found to give rise to a number of unusual optical effects [11-13]. They have also been founed to interact with organic molecules [14] and predicted facilitate catalysis $[15,16]$. These occur effects on a planar TI surface [17-19]. They can be magnified in nanoparticles where both optical and catalytic properties are usually enhanced. The nanoparticles are also favoured by the need for miniaturisation and it is unfortunate that unlike nanowires $[9,20]$ they have been largely overlooked so far.

Metallic nanoparticles differ strongly from bulk metals [21]. Their large surface-to-volume ratio also has consequences for TI nanoparticles. Previously clean TI nanoparticles were predicted to possess surface states with non-isotropic spin and charge distributions [22]. (a)

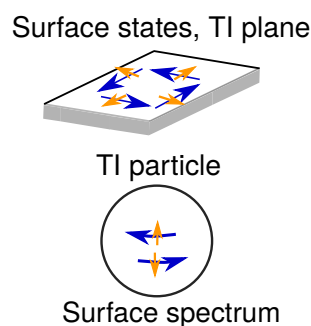

(c)

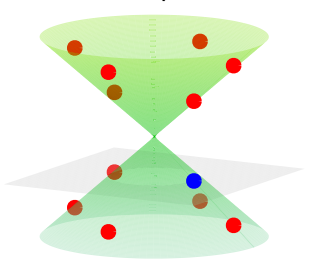

(b)

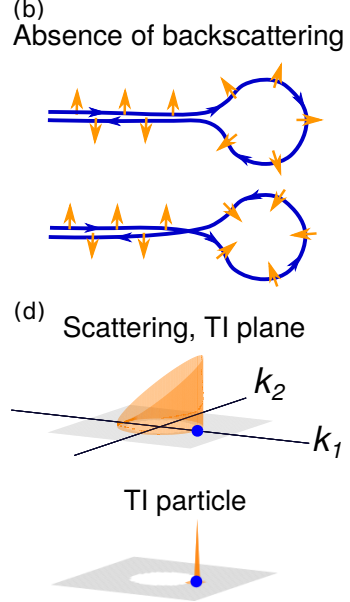

Figure 1. Properties of topologically protected surface states. (a) Momentum (blue arrow) and spin (orange arrow) are locked. (b) The locking protects them from elastic backscattering because the two possible paths interfere destructively. (c) Dirac cone of a planar surface (green) turns into discrete states in nanoparticles (red dots). (d) Illustration of the main result: the discrete states also cannot backscatter elastically and have the same level of protection from disorder as their continuous counterparts.

Moreover, these states give rise to an extra excitation due to coupling of electrons, phonons and light [23]. The latter can be tuned with Fermi level providing an additional degree of freedom when making a metamaterial from such nanoparticles. The delocalised states should also support covalent interaction over long ranges which may be useful in chemistry. The above applications rely on the surface states of TI nanoparticles being robust against disorder. For continuous spectrum on a planar surface the surface states were found to be robust for disorder potentials with amplitudes smaller than the bulk band gap $[24,25]$. This is to be contrasted with the nanoparticles where the surface Dirac cone is no longer continuous but discrete as seen in Fig. 1(c). Our 
main finding is that these discrete states benefit from the same level of protection as in the case of an infinite plane. To show this we first use an analytical model [22] to argue that the states on a clean surface are also protected from elastic backscattering as summarized in Fig. 1(d). Then we add disorder to the surface and study its effects. This can be done using the analytical model [26] or electronic structure simulations. We use the latter route as it allows to resolve details at atomistic level, is not restriected to an ideal sphere and allows to consider all bulk and surface states - not just a few surface ones. Here we use the tight-binding model of Fu-Kane-Mele [8] to study the localization of the surface states in TI nanoparticles for the first time. Previous tight-binding studies of TI nanostructures employed coarse graining based on the slowly-varying envelope approximation $[22,27,28]$ and did not touch upon disorder. Our main finding is that the surface states in TI nanoparticles only become localized when the disorder potential amplitude exceeds the bulk gap. The potentials of this strength can couple the surface with the bulk whereas weaker disorder hardly affects the states.

TI planar surface. Time-reversal symmetry is present when there are no magnetic impurities in the system. In this case the topologically protected states are robust against a certain degree of disorder. To see this it is instructive to study a planar surface first. In this case the topologically protected states appear as an odd number of Dirac cones in the surface bandstructure. Importantly, each cone is not spin-degenerate. Moreover, the corresponding states are spin-momentum locked meaning that the states with opposite momenta have opposite spins. As a result a non-magnetic disorder potential which cannot flip the spin couples different surface states within a cone much less than in spin-degenerate bands. In particular, any state originating from such a Dirac cone cannot be backscattered. The latter follows from the $\pi$ Berry phase acquired by an electron as when going around the cone. To illustrate this we consider here an analytical solution. Such solutions exist for a number of TIs but for concreteness here we consider $\mathrm{Bi}_{2} \mathrm{Se}_{3}$ which possesses a single Dirac cone. To obtain the surface wavefunctions one can solve the four-band $\mathbf{k} \cdot \mathbf{p}$ Hamiltonian valid close to $E=0$, the Dirac point [29]. The solution takes the form $|\psi\rangle=c_{+}|+\rangle+c_{-}|-\rangle$ where $| \pm\rangle$ are the two basis states with opposite angular momenta and the coefficients which depend on the in-plane momentum $k, \mathbf{c}=\left(c_{+}(k), c_{-}(k)\right)^{T}$. The absence of elastic backscattering follows from the fact that $|+\rangle$ and $|-\rangle$ are related by the time-reversal operation $\Theta$. Namely, $\Theta|+\rangle=e^{i \alpha}|-\rangle$ and $\Theta|-\rangle=e^{i \beta}|+\rangle$ where $\alpha-\beta= \pm \pi$ due to the fundamental requirement that $\Theta^{2}=-1$ for fermions. Furthermore, the following relation holds for the two basis states [30]:

$$
\langle a|\Theta| b\rangle=-\langle b|\Theta| a\rangle
$$

where $a, b= \pm$. Let us now consider a general potential,
$V$ due to non-magnetic impurities or defects. Being nonmagnetic this potential is time-reversal symmetric and satisfies

$$
\Theta V \Theta^{-1}=V, \quad V^{\dagger}=V
$$

Using Eqns. 1 and 2 it is then easy to show that

$$
\langle+|V|-\rangle=-\langle+|V|-\rangle=0
$$

Note that we made no assumptions about the spatial form of $V$. For such a generic case elastic backscattering between two surface states is only strictly forbidden, i.e. $\left\langle\psi|V| \psi^{\prime}\right\rangle=0$, when their coefficients are orthogonal $\mathbf{c}^{\dagger} \mathbf{c}^{\prime}=0$. For the surface states below the Dirac point $\mathbf{c}=\left(1, i e^{i \chi}\right)^{T} / \sqrt{2}$ where $\chi=\arg \left(k_{x}+i k_{y}\right)$. The scattering probability is then proportional to $\left|\mathbf{c}^{\dagger} \mathbf{c}^{\prime}\right|^{2}=$ $\left[1+\cos \left(\chi-\chi^{\prime}\right)\right] / 2$ which is shown in Fig. $1(d)$. It implies that disorder potentials with small amplitudes have difficulty localizing the surface states which couple weakly to each other. The localization only occurs when the amplitudes are large enough to couple the surface states to the bulk ones. The same happens in TI particles.

Quantum confinement in all three directions makes the energy spectrum of a TI particle discrete. The surface states for a spherical $\mathrm{Bi}_{2} \mathrm{Se}_{3}$ particle were derived by Imura et al. [22] and confirmed with the tight-binding simulations specific for this material $[22,23]$. The states are delocalised over the surface of the particle with energies given by

$$
E= \pm \frac{\Lambda_{\text {soc }}}{r_{\mathrm{P}}}(n+|m|+1 / 2)
$$

where $\Lambda_{\text {soc }}$ is represents the spin-orbit coupling strength within the model and $r_{\mathrm{P}}$ is the particle's radius. The two quantum numbers: $n=0,1, \ldots$ and $m= \pm 1 / 2, \pm 3 / 2 \ldots$ imply the degeneracy of the states grows as $2,4,6 \ldots$ away from $E=0$ (Fig. 1(c)). From the analytical wavefunctions of the states it follows that they are also protected against backscattering. For example, the doublydegenerate states below $E=0$ with $n=0, m= \pm 1 / 2$ have coefficients, $\mathbf{c}_{m=1 / 2}=(\cos \theta / 2, \sin \theta / 2)^{T} / \sqrt{4 \pi}$ and $\mathbf{c}_{m=-1 / 2}=(\sin \theta / 2,-\cos \theta / 2)^{T} / \sqrt{4 \pi}$ which give $\mathbf{c}_{m=-1 / 2}^{\dagger} \mathbf{c}_{m=1 / 2}=0$ as shown in Fig. 1(d). Thus while considering only first order scattering events (known as the Born approximation) the states in the spherical particle behave similarly to those on the planar surface. Thus we would expect the discrete states to be robust against disorder potentials with amplitudes smaller than the bulk gap. This conclusion agrees with the tight-binding calculations for disordered TIs below. Tight-Binding simulation of a TI slab. We employ the $\mathrm{Fu}$-Kane-Mele model [8] which was previously used to study topological insulator slabs $[8,31]$ and nanowires $[27,28]$. It is not restricted like other tight-binding models used to date $[24,25]$. Unlike those, the Fu-Kane-Mele model does not rely on low-energy (slow spatial variation) approximation. This is a prototypical microscopic 


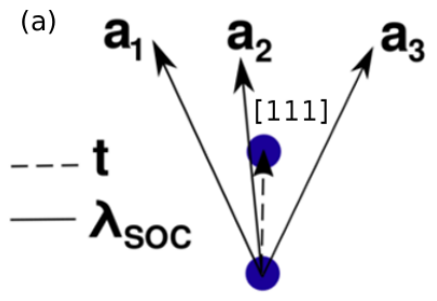

(b) $\quad V_{D}=0$

(c)

$V_{\mathrm{D}}=0.75 E_{\mathrm{G}}$

(d)

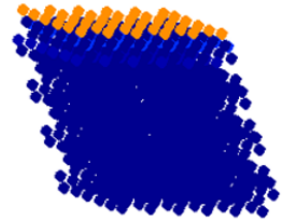

[111]

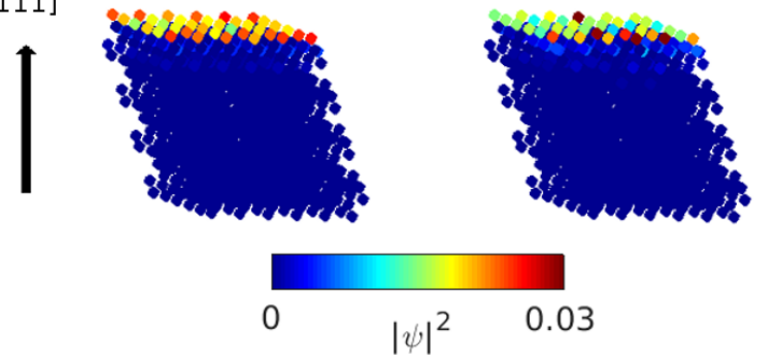

Figure 2. Surface states of a disordered TI slab (a) Unit cell of the Fu-Kane-Mele model TI for the diamond structure. The dashed line stands for sublattice vector for one of four the nearest-neighbor hoppings. Second nearest-neighbor hoppings between atoms separated by a lattice vector are due to spin-orbit coupling. (b) Surface state of a TI slab obtained with the tight-binding model. The surface state at the $\Gamma$-point is shown. (c) It is weakly affected for disorder potentials with amplitudes below the bulk gap and (d) becomes localized for stronger ones. The wavefunction is normalized to the supercell shown. The slab is perpendicular to the [111] direction.

model that displays generic properties of TIs. It consists of atoms on a diamond lattice each carrying one spin-degenerate s-orbital. The Hamiltonian for this elemental TI is given by

$$
H=t \sum_{\langle i, j\rangle} c_{i}^{\dagger} c_{j}+\frac{\lambda_{\mathrm{soc}}}{4 a^{2}} \sum_{\langle\langle i, j\rangle\rangle} c_{i}^{\dagger} c_{j} \sigma \cdot\left(\mathbf{d}_{1} \times \mathbf{d}_{2}\right)
$$

where the first term corresponds to nearest-neighbor hoppings of strength $t$ which occur between the two sublattices in a diamond lattice shown in Fig. 2(a). The other term is due to spin-orbit coupling and includes second-nearest-neighbor hoppings. This term is direction dependent via $\mathbf{d}_{1}$ and $\mathbf{d}_{2}$ which are the two bonds an electron passes on its way from atom $i$ to atom $j . \lambda_{\text {soc }}$ is the spin-orbit coupling constant and $\sqrt{2}\left|\mathbf{a}_{i}\right|=a=1$ is the size of the cubic cell. The topological gap is opened via shortening the nearest-neighbor bond along [111] for this bond $t \rightarrow t+\delta t$. We use $t=\lambda_{\text {soc }}$ and $\delta t=0.4 t$. With these parameters there is a bulk gap, $E_{\mathrm{G}}=2 \delta t$ and the material is a strong TI [8]. The tight-binding Hamiltonian in Eq. 5 does not describe a particular TI. Nevertheless, it serves as a useful prototypical microscopic model. It was also successfully used in the past to simulate energy dispersion in nanowires made of existing TIs [27, 28]. In addition, we find many similarities with the analytical model predictions for the spherical particles [22] as will be seen below.

We consider a slab supercell of size $6 \times 6$ primitive cells in the plane of the slab and 8 cells in the non-periodic, [111], direction (converged w.r.t. size). We terminate it in such a way that atoms at the bottom are all positioned at lattice points while those at the top surface are shifted by a sublattice vector shown in Fig. 2(a) - see Fig. S1(a) in [32]. As a result one Dirac cone centered at the $\Gamma$ point occurs (on each surface). The surface state seen in Fig. 2(b) decays exponentially into the bulk. The surface can become disordered due to adsorbates and point defects. We model the disorder by confining it to one unit cell below each surface. Disorder of strength $V_{\mathrm{D}}$ enters the Hamiltonian (5) as a random on-site potential drawn from the uniform distribution $\left[-V_{\mathrm{D}}: V_{\mathrm{D}}\right]$ which is an established procedure to study localization $[33,34]$. The states become localized only when the disorder reaches $V_{\mathrm{D}}=E_{\mathrm{G}}$ as seen in Fig. 2(c-d). This is representative of the statistically averaged results by Soriano et al. over many disorder realizations. Similar findings have been reported previously with other tightbinding models of TI slabs $[24,25]$. The protection is strongest for the states at the $\Gamma$-point (shown) as they lie deepest in the bulk gap. The same occurs for a TI nanoparticle as shown below. (a)

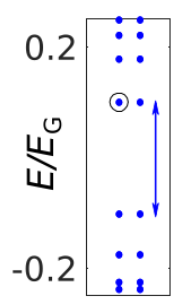

(d)

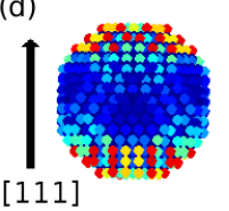

(b)

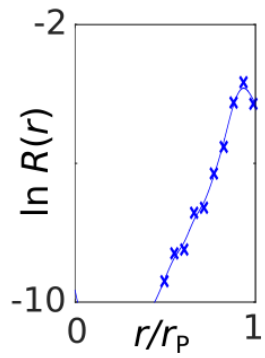

(e)

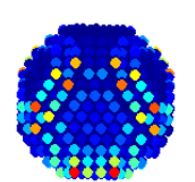

(c)

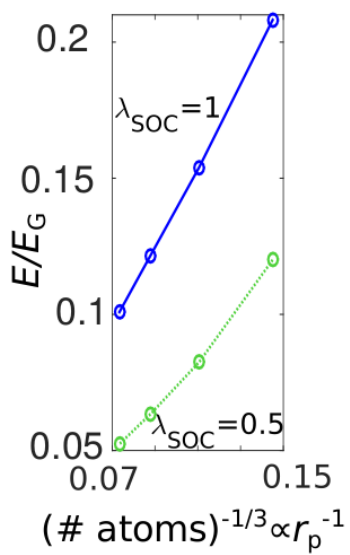

Figure 3. Surface states of a clean TI particle obtained with tight-binding. (a) The energy spectrum near the centre of the bulk gap. Larger spacing between the states close to $E=0$ (marked with an arrow) agrees with the analytical model (see text). (b) Radial wavefunction of the encircled state decays exponentially into the bulk. (d) Probability density of the encircled state. (c) The energy of the state encircled in (a) is proportional to $\lambda_{\mathrm{soc}} / r_{\mathrm{p}}$ also agreeing with the model. (e) Probability density of the first state above $E=0$ when the shorter bond is along [1111] direction (see main text).

Tight-Binding simulation of a TI particle. Topolog- 
ical surface states are a consequence of the bulk bandstructure. Therefore there needs to be enough bulk material to support them. The decay length of topological surface states is about of $1 \mathrm{~nm}$ [23]. As a result, in TI slabs with thickness below $5 \mathrm{~nm}$ the edge states from opposite surface start overlapping and are said to become gapped out [35]. Analogously, a very small particle will have no states in the gap as predicted by Eq. 4. We consider a spherical particle consisting of 2500 atoms. The particle considered is large enough to display topological properties. This follows from the fact there are many surface states within the bulk gap as seen in Fig. 3(a). In addition, surface states decay exponentially and have very little probability density close to the middle of the particles as shown in Fig. 3(b). Besides, we also checked that the results hold for a smaller particle (1500 atoms). We carve the particle out of bulk in such a way that the primitive unit cells remain intact allowing us to retain the inversion symmetry (Fig. $\mathrm{S} 1$ (b) in [32]). Close to the Dirac point, $E=0$, the simulated spectrum of the clean particle consists of doubly degenerate states as shown in Fig. 3(a). The spin-orbit coupling term breaks the sublattice symmetry of the spectrum about $E=0$. The energies of the surface states obtained with the Fu-Kane-Mele model used scale as $\lambda_{\text {soc }} / r_{\mathrm{p}}$ as seen in Fig. 3(c). This has been predicted by the analytical model for spherical particles in Eq . 4 [22]. This suggests that such scaling of the energies is a generic property of TI nanoparticles. Moreover, we calculated the radial wavefunction in Fig. 3(b) by summing $|\psi|^{2}$ over all atoms at a given radius from the particle. The surface states are confined to the boundary and decay exponentially into the bulk - see Fig. 3(b), which also agrees with the analytical model [17, 22]. The states themselves are delocalised over the poles as seen in Fig. 3(d). For smaller particles the states cover a larger fraction of the surface [32]. Apart from size there are two factors which affect the surface wavefunctions through anisotropy. Firstly, there is anisotropy introduced because we cannot carve out a perfect sphere. Secondly, the four nearest-neighbor bonds are not all equivalent - as mentioned earlier, the hopping along [111] direction is stronger. This effect is illustrated in Fig. 3(e) where another bond, along [1̄11], was chosen to be shorter instead (for particles of other shapes see Fig. S2 in [32]). The surface states now occur across two opposite sides. They are modified but still present which highlights their topological nature.

We proceed to study the effects of disorder by adding a random on-site potential to the atoms on the surface as we did for the slab above. Interestingly, even for $V_{\mathrm{D}}=E_{\mathrm{G}}$ the spectrum retains much of its structure close to $E=0$ in Fig. 4(a) (for a representative disorder realization - ensemble results can be found in Fig. S3 of [32]). This is surprising given that we add the disorder potential to all atoms outside radius $r_{\mathrm{D}}$ in Fig. 4(b)
(700 of the total 2500). In the clean case a state close to the Dirac point has enhanced probability density at the poles which lie on the [111] direction. The large energy difference between the states is rather robust as shown in Fig. 4(c). It stays almost constant and shows very small spread up to $V_{\mathrm{D}}=E_{\mathrm{G}}$ which may be interesting for applications in optics. This energy difference disappears for larger $V_{\mathrm{D}}$ that can couple bulk and surface states localizing the latter - just as in the slab case. (a)

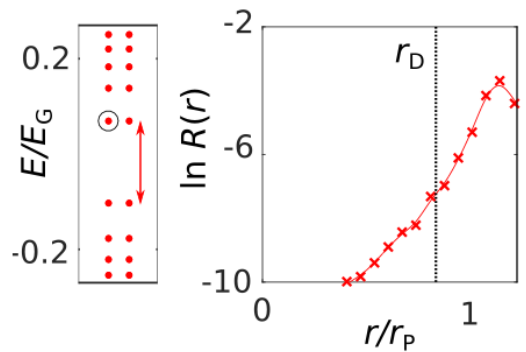

(c)

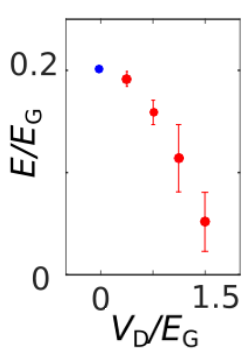

Figure 4. Surface states of a TI particle obtained with tightbinding. All surface atoms are disordered (see text). (a) The energy spectrum near the centre of the bulk gap. (b) The radial wavefunction of the state encircled in (a) for disorder strength $V_{\mathrm{D}}=E_{\mathrm{G}}$. All atoms with $r>r_{D}$ carry the disorder potential. (c) Difference in energy for the states marked with an arrow in (a). A mean over 25 disorder realisations for each value of $V_{\mathrm{D}}$ is shown; error bars denote the standard deviation of the sample.

It is interesting to compare how disorder affects particles of different shapes. Localization is difficult to quantify using conventional measures, such as the inverse participation ratio, designed for infinite systems $[25,31]$ (it also requires imposition of an arbitrary cut off away from the surface) because the eigenstates of the undisordered particles also have non-uniform probability densities. To circumvent this we define a function, $s_{\mathrm{D}}$, that measures the deviation from inversion symmetry due to disorder. For a given state, $\psi$, it is given by

$$
s_{\mathrm{D}}=\left.\left|\sum_{i \in \text { atoms }}\right| \psi_{i}\right|^{2} \hat{\mathbf{r}}_{\mathbf{i}} \mid
$$

where $\hat{\mathbf{r}}_{\mathbf{i}}$ is the unit vector towards the position of an atom $i$. With the origin at the particle's centre, we have $s_{\mathrm{D}}=0$ in the clean case. In the strong disorder case it may reach the value $s_{\mathrm{D}}=1$ meaning that the state is localized on one atom or several of them in the same radial direction. Any pathological charge distributions are taken care of by statistically averaging over many realizations with a given disorder strength, $V_{\mathrm{D}}$. The results for the sphere with surface disorder discussed earlier are presented in Fig. 5(a). They show that $s_{\mathrm{D}}$ increases with $V_{\mathrm{D}}$ and saturates close to $V_{\mathrm{D}}=E_{\mathrm{G}}$. For stronger disorder the wavefunction becomes localized on one of the poles - see Fig. 5(b). Also, the large energy difference between the states immediately above and below $E=0$ disappears as seen in Fig. 4(c). We have also 
checked that $s_{\mathrm{D}}$ increases more rapidly with $V_{\mathrm{D}}$ as we decrease $\lambda_{\text {soc }}$ approaching trivial insulator (topological transition is driven by large $\lambda_{\text {soc }}-$ see Fig. S4 in [32]). In addition, we have studied the bulk disorder (pick only the atoms with $r<r_{D}$ in Fig. 4(b) and add disorder to them). Because the states are confined to the surface they are practically unaffected for $V_{\mathrm{D}}$ values considered as seen from Fig. 5(b). Stronger bulk disorder of the order of the band width localizes the states by destroying the bulk bandstructure that supports them. This was also found in the previous studies on TI slabs [24, 31]. The protection described here also holds for particles of other shapes considered below.

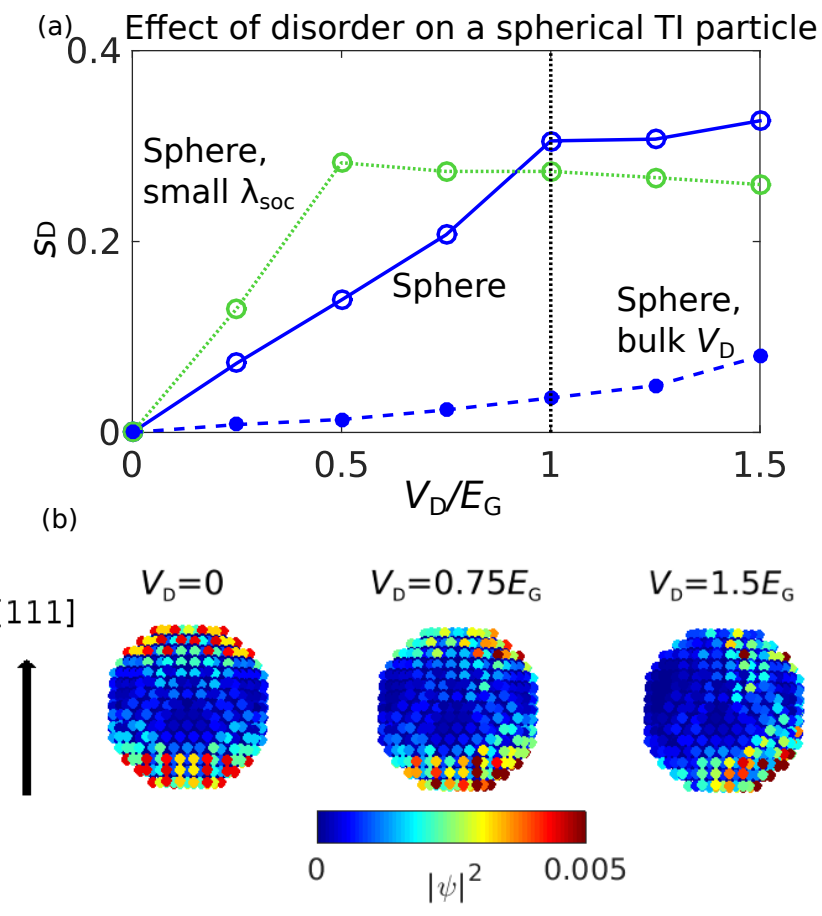

Figure 5. Symmetry deviation, $s_{\mathrm{D}}$, as a function of disorder strength, $V_{\mathrm{D}}$ (see text) for the first state above $E=0$ (a) Solid blue lines with empty circle (square) correspond to sphere (cube) with the surface fully disordered, green line - to a sphere with smaller spin-orbit coupling $\left(\lambda_{\text {soc }}=0.5\right)$ which is closer to a trivial insulator, dashed line with solid circles - to a sphere with bulk disorder. Each data point represents an average over 25 disorder realizations; errors are smaller than the marker sizes. (b) Probability distribution of the first surface state above $E=0$ on the spherical topological nanoparticle; [111] direction is upwards.

The Fu-Kane-Mele model employed allows us to model structural disorder by making the surface more rough. To do this we position more atoms on the otherwise smooth surface. We take a clean spherical particle (1500 atoms) and add additional atoms (400) to it randomly. The atoms are added in pairs (unit cells) as discussed in the Figs. S5-7 of [32]. Adding atoms weakly affects the states slightly decreasing the spacing between them as the effective particle's radius grows (see Fig. S5). Next we add on-site disorder potential to the atoms furthest from the centre of the particle (700 out of the total 1900). The only difference from the smooth sphere is that the states become slightly more prone to disorder as shown in Fig. 6(a). Still the states remain extended unti $V_{\mathrm{D}}=E_{\mathrm{G}}$ as can be seen in Fig. 6(b).
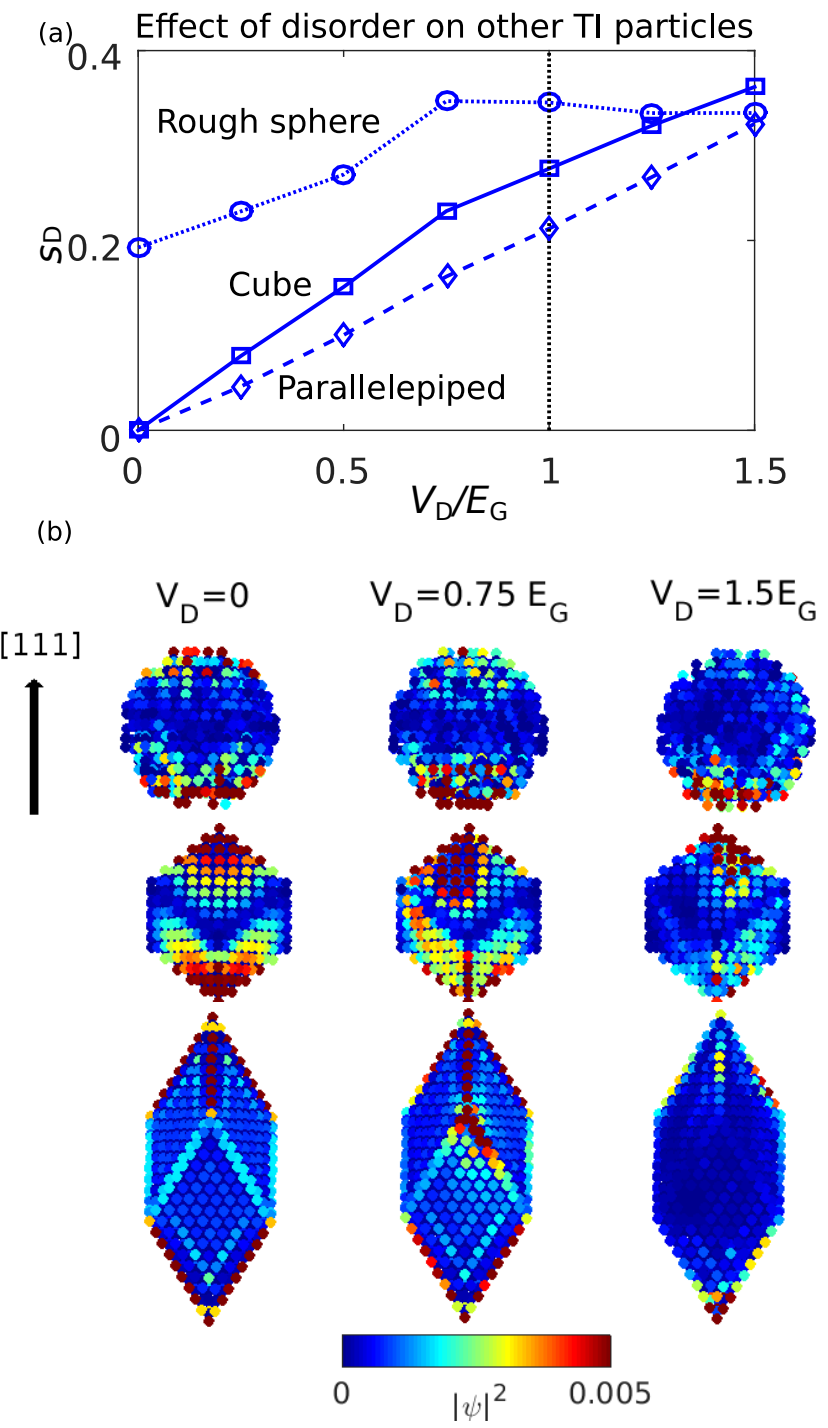

Figure 6. Symmetry deviation, $s_{\mathrm{D}}$, as a function of disorder potential strength, $V_{\mathrm{D}}$ (see text) for the first state above $E=0$ (a) Dotted line with empty circles corresponds to a sphere with rough surface, solid line with squares - to a cubic particle, dashed line with diamonds - to a parallelepiped. The latter is the most natural shape for the tight-binding model employed. Each data point represents an average over 25 disorder realizations; errors are smaller than the marker sizes. (b) Probability distribution of a surface state on the nanoparticles mentioned above for different disorder potential strengths; [111] direction is upwards.

Like surface roughness the particle's shape has also little effect on the protection of the states. This can be seen in Fig. 6(a) where the disorder function, $s_{\mathrm{D}}$ is calculated for a cubic particle (600 atoms disordered out of 1300 total). The results are similar to those for the spherical particle in Fig. 5, although $s_{\mathrm{D}}$ now 
plateaus at $V_{\mathrm{D}}=1.5 E_{\mathrm{G}}$. Furthermore, this behavior is closely followed by a parallelepiped particle. The latter is the most natural shape for the model employed. As can be seen from Fig. 6(a) it shows the strongest protection against localization by disorder (all 2000 atoms are disordered). The probability densities for the cubic and parallelepiped case and their reaction to disorder are shown in Fig. 6(b). These cases also illustrate how the shape of the particle modifies the surface states depending on which closed packed planes (perpendicular to nearest-neighbor bonds) are present.

Conclusions We have shown that the surface states in TI nanoparticles possess the same level of protection as in the case of an infinite surface. These states survive disorder with extensive coverage (affecting all surface atoms) and disorder potential strength spanning two bulk band gaps. This has been shown using tightbinding simulations for particles of spherical, cubic and parallelepipedal shapes. Moreover, we have shown that these results also hold in the case of rough surface. The protection of the small particles studied here draws a bridge to the protection in bulk samples with planar surfaces. It suggests that all TI particles with intermediate sizes also have protected states. Such protected delocalized states may find use in chemical catalysis. Moreover, the robust spectrum of the nanoparticles made of currently available TIs has appropriate energy [23] for applications in $\mathrm{THz}$ spectroscopy and nanophotonics. Finally, the discretization of the states should reduce scattering by phonons. This "phonon bottleneck" effect was never observed in quantum dots [36] due to scattering via surface channels [37] so topological nanoparticles provide a new avenue to explore it.

The authors thank Max Boleininger for useful discussions. G.S. was supported through a studentship in the Centre for Doctoral Training on Theory and Simulation of Materials at Imperial College London funded by EPSRC Grant No. EP/L015579/1.

[1] C. L. Kane and E. J. Mele, Phys. Rev. Lett. 95, 146802 (2005).

[2] M. Z. Hasan and C. L. Kane, Rev. Mod. Phys. 82, 3045 (2010).

[3] X.-L. Qi and S.-C. Zhang, Rev. Mod. Phys. 83, 1057 (2011).

[4] F. D. M. Haldane and S. Raghu, Phys. Rev. Lett. 100, 013904 (2008).

[5] L. Lu, J. D. Joannopoulos, and M. Soljačić, Nature Photon. 8, 821 (2014).

[6] E. Prodan and C. Prodan, Phys. Rev. Lett. 103, 248101 (2009).

[7] N. Goldman, G. Juzeliunas, P. Ohberg, and I. B. Spielman, Rep. Prog. Phys. 77, 126401 (2014).

[8] L. Fu, C. L. Kane, and E. J. Mele, Phys. Rev. Lett. 98, 106803 (2007).

[9] S. S. Hong, Y. Zhang, J. J. Cha, X.-L. Qi, and Y. Cui,
Nano Letters 14, 2815 (2014).

[10] P. Roushan, J. Seo, C. V. Parker, Y. S. Hor, D. Hsieh, D. Qian, A. Richardella, M. Z. Hasan, R. J. Cava, and A. Yazdani, Nature 460, 1106 (2009).

[11] D. Hsieh, F. Mahmood, J. W. McIver, D. R. Gardner, Y. S. Lee, and N. Gedik, Phys. Rev. Lett. 107, 077401 (2011).

[12] J. W. McIver, D. Hsieh, H. Steinberg, P. Jarillo-Herrero, and N. Gedik, Nat Nano 7, 96 (2012).

[13] P. Olbrich, L. Golub, T. Herrmann, S. Danilov, H. Plank, V. Bel'kov, G. Mussler, C. Weyrich, C. Schneider, J. Kampmeier, D. Grützmacher, L. Plucinski, M. Eschbach, and S. Ganichev, Phys. Rev. Lett. 113, 096601 (2014).

[14] S. Jakobs, A. Narayan, B. Stadtmüller, A. Droghetti, I. Rungger, Y. S. Hor, S. Klyatskaya, D. Jungkenn, J. Stöckl, M. Laux, O. L. A. Monti, M. Aeschlimann, R. J. Cava, M. Ruben, S. Mathias, S. Sanvito, and M. Cinchetti, Nano Lett. 15, 6022 (2015).

[15] H. Chen, W. Zhu, D. Xiao, and Z. Zhang, Physical Review Letters 107, 056804 (2011).

[16] J. Xiao, L. Kou, C.-Y. Yam, T. Frauenheim, and B. Yan, ACS Catalysis 5, 7063 (2015).

[17] F. Zhang, C. L. Kane, and E. J. Mele, Phys. Rev. B 86, 081303 (2012).

[18] N. Virk and O. V. Yazyev, Scientific Reports 6, 20220 (2016).

[19] J. Wang, Y. Meir, and Y. Gefen, Physical Review Letters 118, 046801 (2017).

[20] D. Kong, J. C. Randel, H. Peng, J. J. Cha, S. Meister, K. Lai, Y. Chen, Z.-X. Shen, H. C. Manoharan, and Y. Cui, Nano Letters 10, 329 (2010).

[21] J. M. Fitzgerald, P. Narang, R. V. Craster, S. A. Maier, and V. Giannini, Proceedings of the IEEE 104, 2307 (2016).

[22] K.-I. Imura, Y. Yoshimura, Y. Takane, and T. Fukui, Phys. Rev. B 86, 235119 (2012).

[23] G. Siroki, D. K. K. Lee, P. D. Haynes, and V. Giannini, Nat. Commun. 7, 12375 (2016).

[24] G. Schubert, H. Fehske, L. Fritz, and M. Vojta, Physical Review B 85, 201105 (2012).

[25] V. Sacksteder, T. Ohtsuki, and K. Kobayashi, Physical Review Applied 3, 064006 (2015).

[26] A. C. Durst, Physical Review B 93, 245424 (2016).

[27] R. Egger, A. Zazunov, and A. L. Yeyati, Phys. Rev. Lett. 105, 136403 (2010).

[28] A. Kundu, A. Zazunov, A. L. Yeyati, T. Martin, and R. Egger, Phys. Rev. B 83, 125429 (2011).

[29] C.-X. Liu, X.-L. Qi, H. Zhang, X. Dai, Z. Fang, and S.-C. Zhang, Phys. Rev. B 82, 045122 (2010).

[30] Y. Ando, J. Phys. Soc. Jpn. 82, 102001 (2013).

[31] D. Soriano, F. Ortmann, and S. Roche, Physical Review Letters 109, 266805 (2012).

[32] See Supplemental Material at [URL will be inserted by publisher] for details of tight-binding simulations and how the particles were constructed.

[33] P. A. Lee and D. S. Fisher, Physical Review Letters 47, 882 (1981).

[34] F. Evers and A. D. Mirlin, Reviews of Modern Physics 80, 1355 (2008).

[35] Y. Zhang, K. He, C.-Z. Chang, C.-L. Song, L.-L. Wang, X. Chen, J.-F. Jia, Z. Fang, X. Dai, W.-Y. Shan, S.-Q. Shen, Q. Niu, X.-L. Qi, S.-C. Zhang, X.-C. Ma, and Q.-K. Xue, Nat Phys 6, 584 (2010). 
[36] D. M. Mittleman, R. W. Schoenlein, J. J. Shiang, V. L. Colvin, A. P. Alivisatos, and C. V. Shank, Physical Review B 49, 14435 (1994).

[37] R. R. Cooney, S. L. Sewall, K. E. H. Anderson, E. A.
Dias, and P. Kambhampati, Physical Review Letters 98, 177403 (2007). 